\title{
Numerical modelling of cathodic protection systems for deep well casings
}

\author{
A. B. Peratta, J. M. W. Baynham \& R. A. Adey \\ CM BEASY Ltd, UK
}

\begin{abstract}
This work is focused on the 3D simulation of cathodic protection (CP) systems for metallic deep well casings immersed in stratified soil. The soil is considered as a multi-layered electrolyte with electric conductivity, which varies from layer to layer. Given a CP system, the aim of the simulation is to predict the level of protection (including the normal current entering the structure) against corrosion along the well casing. It is common practice to fill the annular space between soil and metallic structure with cement along the whole depth. The paper presents a modelling approach which incorporates this feature. In addition, a unique $\mathrm{CP}$ system may be used to protect more than one well. In this case, the simulation is used to analyse problems of interference and load unbalance to the electrical resistance in the power lines and flow lines. The modelling approach is aimed at simplifying the model design, especially for dealing with multi-layered electrolyte, and improving the accuracy over existing traditional techniques, which entail a detailed and time consuming representation in the model of the interfaces between different layers of the electrolyte. The effects of different scenarios of electrolytes on the CP system are considered.
\end{abstract}

Keywords: cathodic protection, Boundary Element Method, deep well casings.

\section{Introduction}

The predictive simulation of cathodic protection (CP) systems applied to well casings provides a number of advantages at the design, monitoring and optimization stages of the project. Accurate modelling capabilities help to gain better understanding of the relationship between different $\mathrm{CP}$ variables, the infrastructure to be protected and its surrounding environment. 
An effective method to monitor corrosion in buried structures is to measure the pipe/soil potential along its span. With horizontal pipelines this is technically feasible; however the access to data on the local CP performance in well casings is very limited and can only be obtained using expensive logging tools.

Deep well casing depths vary usually from a few hundred meters to a few kilometres. Along its depth the casing may penetrate different geological regions with varying physical properties including water content, porosity, permeability, and salinity among others. Therefore, from the modelling point of view, the casing can be considered as exposed to a stratified (multi-layered) electrolyte of varying electrical conductivity. Moreover, from layer to layer the polarization properties of the metallic structure may vary in addition to the characteristic conductivity, due to the corrosivity of the medium.

The electrical conductivity of the soil and its distribution are key parameters, which determine how current flows between the anode and the metallic structure, and therefore strongly influence the levels of corrosion protection along the casing. The modelling of $\mathrm{CP}$ systems involves predicting the current and potential fields at any point in the electrolyte and at surfaces of electrodes. Under most common situations, this requires solving the steady state charge conservation equation in the electrolyte in $3 \mathrm{D}$ space given by: $\nabla \cdot \mathbf{j}=0, \mathbf{x} \in \Omega$ where $\mathbf{j}=-\sigma(\mathbf{x}) \nabla V_{e}(\mathbf{x})$ represents current density, $\sigma$ is the electrolyte conductivity, $\mathrm{Ve}$ is the potential in the electrolyte measured against remote earth, and $\Omega$ represents the integration domain (electrolyte).

The Boundary Element Method (BEM) [1] has been widely used to solve Laplacian equations and in particular simulate cathodic protection systems for underground and offshore structures [2-6]. The most significant advantages of the method are first that the formulation is based on the fundamental solution of the leading partial differential operator in the governing equation, and second that it requires only mesh discretisation on the boundaries of the problem. The former aspect confers high accuracy, while the latter substantially simplifies the pre-processing stage of the model, since volume discretisation is not needed. The standard BEM is traditionally aimed at solving homogeneous electrolytes. In case of non-homogeneous conditions it is common practice to combine BEM with domain decomposition or multi-region (MR) technique. In this way the electrolyte, considered as piecewise homogeneous, is represented as a collection of sub-regions, each one of them with homogeneous conductivity. Then, neighbouring regions are connected to each other by prescribing continuity of potential and normal current density through their common interface [3].

In this paper a new technique for computing accurately and efficiently 3D problems involving deep well casings immersed in multilayered soil is used and compared against the traditional MR. The goal of the Multi Layer (ML) approach is to avoid including in the model the interfaces between different layers, by employing a fundamental solution specifically designed for multilayer materials. The major advantages over the more traditional MR approach are mainly the reduction in the engineering time to prepare a model, since interfaces do not need to be included in the model. Also the solving time is minimised, since only the degrees of freedom representing the well casing and the anodes 
need to be considered. An important consequence of this feature is that the number of layers will not significantly affect the computational cost of the calculation. Moreover, a common limitation in BEM is that the distance between two elements of the mesh must not be too small in comparison to the characteristic length of the largest element, otherwise accuracy and stability of the solution is compromised. This translates into a practical limitation in modelling thin layers of electrolyte, or models in which the thickness of the layer is small in comparison with its lateral extension. However, the ML approach does not suffer from this limitation, and therefore allows the end user to include thin layers without major problems of accuracy or high computational cost.

The paper is organised as follows: Section 2 provides briefly some background information for the ML. Section 3 presents a comparison between MR and ML results in a particular case scenario. Section 4 demonstrates the modelling capabilities of subsurface thin layers. This type of modelling is difficult and time consuming with the standard MR approach, but rather straightforward to conduct with the ML. Section 5 presents a method for modelling the cement in the annular space between the outer tube and soil. Section 6 presents the case of a single anodic bed protecting two distant casings. Overall conclusions are given in Section 7.

\section{Multi layer modelling approach}

The modelling of $\mathrm{CP}$ systems protecting well casings is intrinsically three dimensional (3D). Hence 3D BEM kernels are required. The ML approach incorporates the stratified nature of the medium into the corresponding Green's function. Then BEM is applied in the same way as in the case of the homogeneous electrolyte, except that the Green's function for the homogeneous Laplace equation given by $1 /(4 \pi r)$ is replaced by the multi-layer Green's function given by:

$$
G\left(\mathbf{x}_{i}, \mathbf{x}_{j}, m, n\right)=\frac{1}{4 \pi \sigma_{m}} \sum_{k=1}^{N \exp } \frac{\alpha_{i j m l}}{\left\|\mathbf{x}_{i}-\mathbf{x}_{j}+\mathbf{g}_{i j}\right\|}
$$

where $\mathbf{x}$ denotes the $3 \mathrm{D}$ coordinates, the sub indices $\mathbf{i}$ and $\mathbf{j}$ stand for the source and field point, respectively; $\mathrm{m}$ and 1 indicate the layer of the source and field points, respectively; $\alpha_{i j m l}$ is a weight coefficient and $\mathbf{g}_{i j m l}$ denotes a displacement vector. The calculation of the weight and displacement vectors goes beyond the scope of this paper and can be derived from ref $[2,3,7-9,11]$ and references therein. Finally, the Green's function (5) replaces the 1/r kernel used for homogeneous regions, and employs any standard BEM strategy. In this case, collocation direct BEM has been adopted.

\section{Case study: comparison between MR and ML}

A number of comparison tests and validations of the ML have been performed using as reference the MR technique. This section introduces a conceptual model 
used to compare the results and calculation performance of both methods. The conceptual model consists of a single well casing and one ICCP anodic bed in stratified soil consisting of 7 layers. The well is $1750 \mathrm{~m}$ deep and consists of 4 sections of different diameter. The specification of the main metallic tube relevant to the simulation is shown in Table 1.

Table 1: $\quad$ Main tube specifications.

\begin{tabular}{|l|l|l|l|l|l|l|}
\hline Section & Soil layer & Zmin & Zmax & $\mathbf{D}_{\text {ext }}$ & $\mathbf{t}$ & $\mathbf{R}$ \\
\hline & & $\mathrm{m}$ & $\mathrm{m}$ & $\mathrm{m}$ & $\mathrm{m}$ & $\mathrm{Ohm} / \mathrm{m}$ \\
\hline 1 & 1 & 0 & 300 & 0.35 & 0.012 & $1.37 \mathrm{E}-05$ \\
\hline 2 & 2 & 300 & 500 & 0.25 & 0.012 & $1.94 \mathrm{E}-05$ \\
\hline 3 & $2,3,4$ & 500 & 800 & 0.175 & 0.012 & $2.83 \mathrm{E}-05$ \\
\hline 4 & $4,5,6,7$ & 1200 & 1750 & 0.15 & 0.012 & $3.34 \mathrm{E}-05$ \\
\hline
\end{tabular}

Figure 2 (right) illustrates the conceptual model. The numbers on the left hand side indicate the conductivity of each layer, while the scale on the right hand side indicates the $\mathrm{z}$ coordinate of each interface. The dot circle labelled as "O" in the figure represents the origin of the XYZ system of coordinates assumed in this work. The anode bed, represented by a $30 \mathrm{~cm}$ diameter by $8 \mathrm{~m}$ long cylinder, is located $75 \mathrm{~m}$ apart from the well in $\mathrm{x}$ direction.

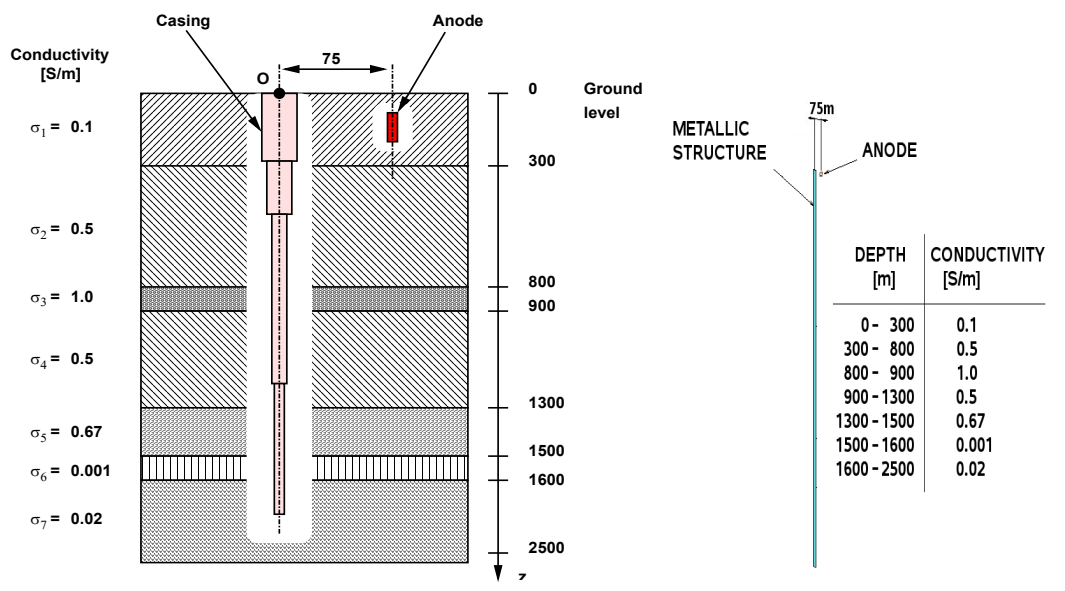

Figure 1: $\quad$ Conceptual model of MR (left). ML model (right).

The size of the linear system of equations that needs to be solved in this case would be $N_{D O F} \times N_{D O F}=3339 \times 3339$, where $N_{D O F}$ is the number of degrees of freedom. The top of the anode is at $30 \mathrm{~m}$ deep and the total current injected into the $\mathrm{CP}$ system is IA $=10 \mathrm{~A}$. The scenario posed for this case study is similar to the one presented in earlier works apart from minor variations. The discretisation mesh of the MR model is shown in Fig 3. The interfaces between layers need to be discretised and incorporated in the model. On the other hand, the ML requires the discretisation of the casing and anode only, thus avoiding elements on the interfaces and bounding box. 


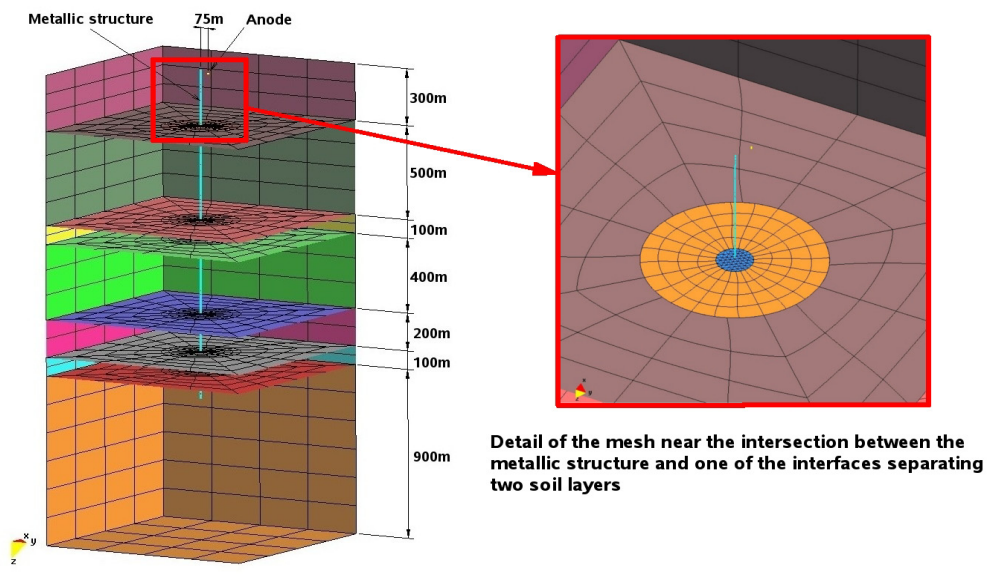

Figure 2: $\quad$ Mesh discretisation for the MR model.

In this example the casing was discretised with 177 tube elements and the anode with 10 elements. Hence, the total number of degrees of freedom is 187 , and the corresponding linear system of equations is of size $187 \times 187$. The ML model is shown in Fig.1 (right). A comparison between MR and ML for the over potential along the structures is shown in Fig 4. More detailed results of the comparison can be found in ref [3].

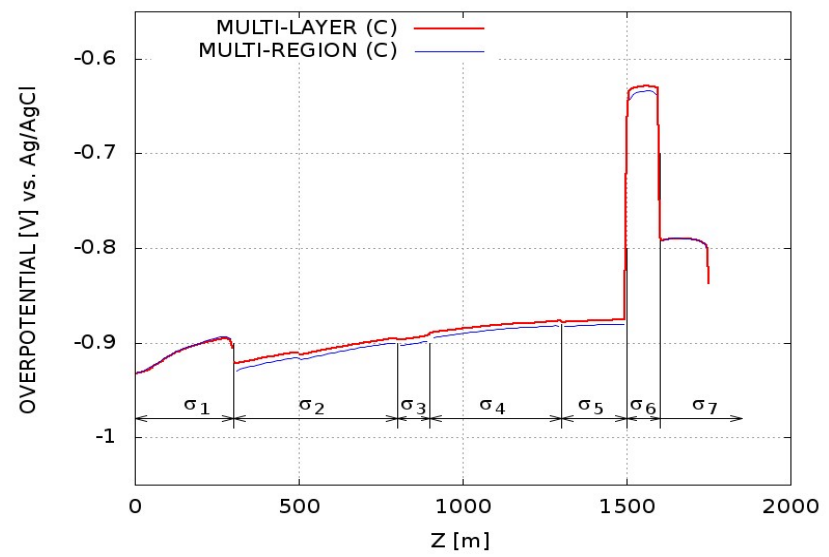

Figure 3: Over potential along depth. Comparison between ML (thick red) and MR (thin blue).

\section{Effect of thin layers}

One of the major advantages of the ML is that it is not limited on the number of layers, horizontal extension or thickness of each layer. In order to illustrate this 
feature and to provide better understanding of the effects of the conductivity of thin layers on the over potential distribution on the whole structure, an uppermost subsurface layer $30 \mathrm{~m}$ thick with variable conductivity has been added to the model. This type of study is more difficult to conduct with the MR approach in view of the fine discretisation needed for the interface of the thin layer. The over potential in function of depth for different sub-surface conductivities in layer 0 is shown on the left of Figure 4. It can be seen that the conductivity of the soil in the first layer does not substantially affect the over potential anywhere else apart from the first $30 \mathrm{~m}$ in contact with that layer. Each curve corresponds to a different soil conductivity in layer 0 which extends from ground level to $30 \mathrm{~m}$. The depth is represented in logarithmic scale, since the main changes of over potential occur in the first few soil layers starting from ground level.

Figure 4 on the right shows the average over potential in the region $0<\mathrm{z}<$ $30 \mathrm{~m}$ of the casing for different electrolyte conductivity of the first layer. Lower conductivity in the subsurface layer translates into more negative over potentials.
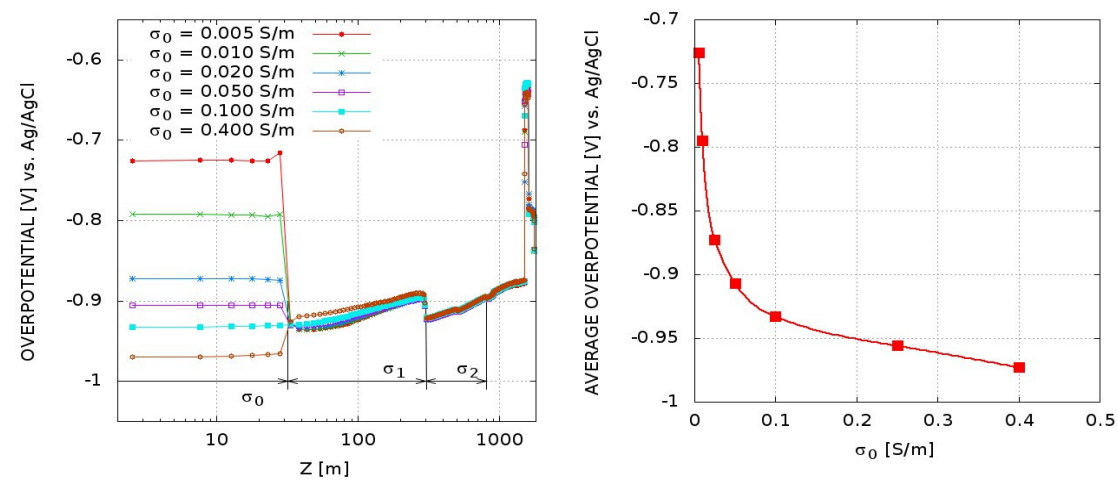

Figure 4: Over potential along the well casing in the 8-layer soil model.

\section{Cement layer}

This section considers the influence of a cement layer surrounding the casing from the electrical conductivity point of view, i.e. any other effect apart from the ohmic drop has not been considered.

Figure 5 (left) shows an arbitrary cross section of the well under consideration. The current density in the concrete is considered to be oriented in radial direction. Under these circumstances, the concrete is introduced into the model as a passive media which introduces an additional ohmic-type voltage drop in the electrolyte. The idea is to derive the equivalent resistance offered by the concrete and use it to adjust the original polarization curve of the steel, as illustrated in Figure 5 (right). The original polarization curve of the steel shown in red is corrected with a linear ohmic drop (dashed line). The resulting polarization curve used in the model is represented with a thick black continuous 
line. Assuming that the current density vector has only radial component, its magnitude at the internal radius $r_{2}$ varies linearly with the voltage drop across the cement according to: $j\left(r_{2}\right)=k_{c} r_{2} \log \left(r_{1} / r_{2}\right) \Delta \phi$, where $\Delta \phi=\phi(r 1)-\phi(r 2)$ is the potential difference across the cement, and $\mathrm{kc}$ is the cement electrical conductivity. For practical convenience, we define the surface distribution of ohmic resistance $\left(\rho_{s}\right)$ as $\rho_{s}=\left(r_{2} / k_{c}\right) \log \left(r_{1} / r_{2}\right)$. Then the over potential (V) of the equivalent polarization contemplating the IR drop across the concrete is corrected according to: $V=V-\rho_{s} j$. In order to investigate the effect of the concrete surrounding the metallic case, the same model of well casing described above was considered in the following scenarios named "BARE", "WET", "DRY" and "MIX".

- "BARE": This scenario considers the case of bare steel (no concrete at all).

- "WET": The metallic case is inside a cement cylinder of external diameter $0.9 \mathrm{~m}$. The concrete is considered highly porous and water has infiltrated.

- "DRY": Same as wet, but with lower conductivity.

- "MIX1": This scenario assumes that the pipe sections 1,2, and 3 are inside a cylindrical column of dry cement, while section $4(1200<\mathrm{z}<$ $1750 \mathrm{~m})$ is considered to be damaged and therefore with the conditions of the wet case.
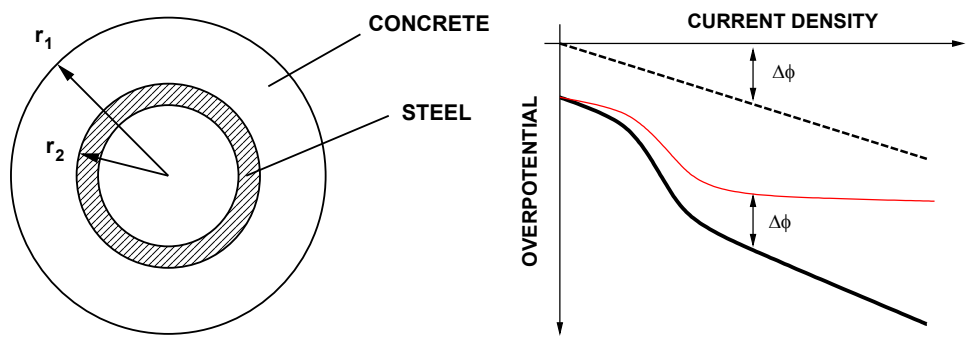

Figure 5: Cross section of the well casing (left). Adjustment of the original polarization curve due to the ohmic drop in the cement (right).

The two types of concrete considered are shown in table 2 .

Table 2: $\quad$ Cement conductivity.

\begin{tabular}{|l|l|}
\hline Type of concrete & Electric Conductivity \\
\hline Wet & $0.025 \mathrm{~S} / \mathrm{m}$ \\
\hline Dry & $0.004 \mathrm{~S} / \mathrm{m}$ \\
\hline
\end{tabular}

The details of each case scenario are shown in table 3 . 
Table 3: $\quad$ Case scenarios.

\begin{tabular}{|c|c|c|c|c|c|c|c|c|}
\hline \multirow{2}{*}{$\begin{array}{l}\text { Sec } \\
\text { tion }\end{array}$} & \multicolumn{2}{|l|}{ Span } & \multirow{2}{*}{$r_{1}$} & \multirow{2}{*}{$r_{2}$} & \multirow[b]{2}{*}{$h=r_{1}-r_{2}$} & \multicolumn{3}{|c|}{$\rho_{s}$} \\
\hline & $\mathbf{Z}_{\min }$ & $\mathbf{Z}_{\max }$ & & & & WET & DRY & MIX1 \\
\hline & m & $m$ & $m$ & $m$ & $m$ & Ohm m2 & Ohm m2 & Ohm m2 \\
\hline 1 & 0 & 300 & 0.45 & 0.175 & 0.05 & 2.87 & 17.95 & 17.95 \\
\hline 2 & 300 & 500 & 0.45 & 0.125 & 0.1 & 2.78 & 17.38 & 17.38 \\
\hline 3 & 500 & 800 & 0.45 & 0.0875 & 0.1375 & 2.49 & 15.56 & 15.56 \\
\hline 4 & 1200 & 1750 & 0.45 & 0.075 & 0.15 & 2.33 & 14.59 & 2.33 \\
\hline
\end{tabular}

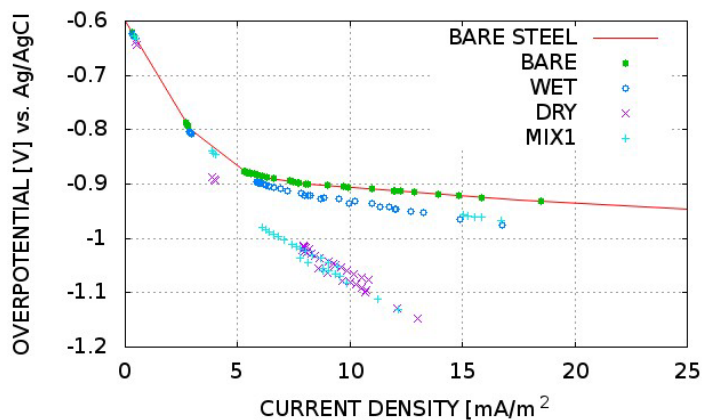

Figure 6: Polarisation curves.
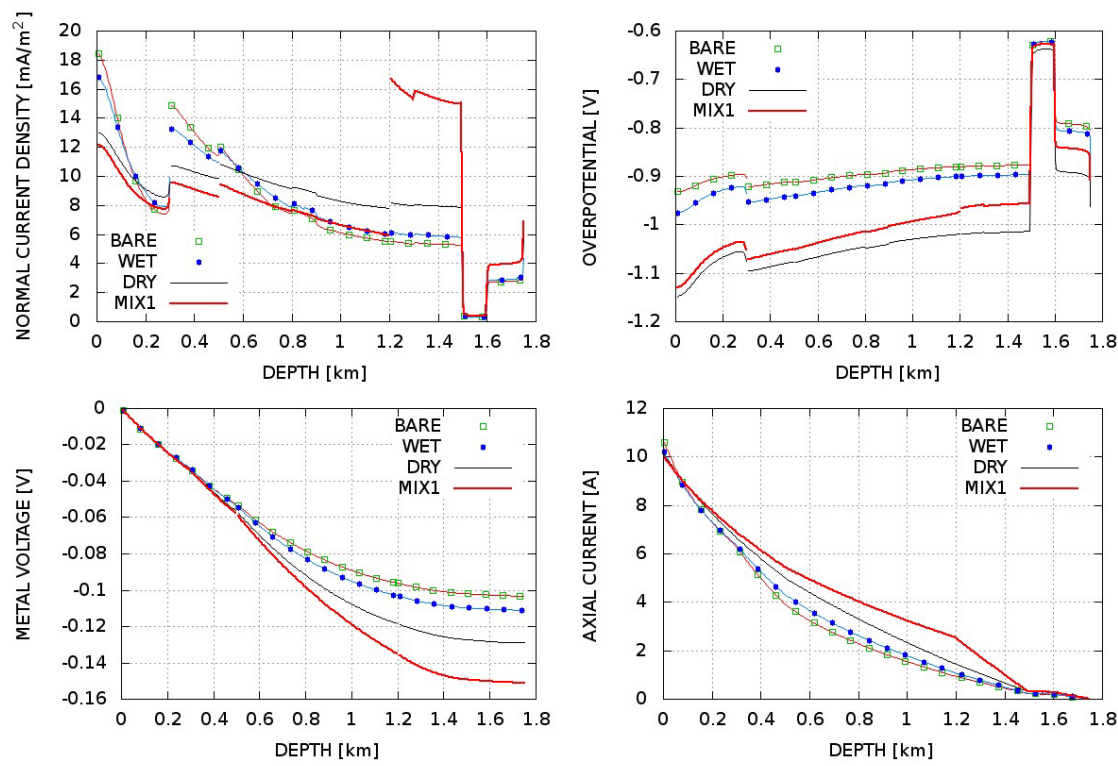

Figure 7: Normal current density (top left), over potential (top right), metal voltage (bottom left) and axial current density (bottom right) in function of depth for "bare", "wet", "dry" and "mix" scenarios. 
The external diameter of the cement lining is kept constant and equal to $0.45 \mathrm{~m}$, hence the thickness of the cement wall (h) varies along depth.

As a consequence the resulting polarization curve is modified with different IR drop resistances depending on the sector of the well considered.

Fig 7 shows a comparison of normal current density, over potential, metal voltage and axial current density for the different scenarios.

These results indicate how the model can be used to investigate different scenarios and conduct "what if" studies. For example, the ohmic drop across

\section{Multiple wells}

The physical situation of this case scenario is shown in Fig 8. The model corresponds to the bare steel case and includes the IR drop in the connections to the power supply but not those in the associated flow lines. The two wells are protected with a single ICCP anode.

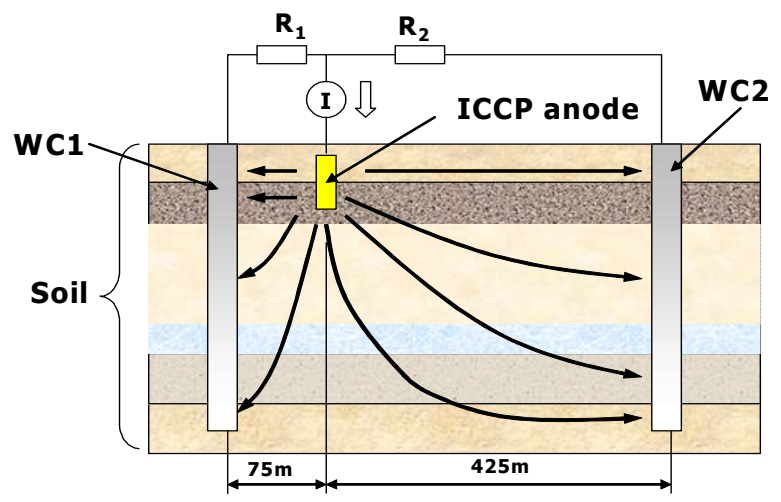

Figure 8: Two well casings connected to a unique ICCP anode.

The anode is $75 \mathrm{~m}$ apart from $\mathrm{WC} 1$ and $425 \mathrm{~m}$ apart from $\mathrm{WC} 2$. The electrical resistance of the power supply cable is represented with the resistors R1 and R2 of 0.4 and $1.44 \mathrm{Ohm}$ respectively.

Therefore the unbalance in the currents flowing to the two wells is not only due to the different resistance in the return path circuit but also due to the ohmic IR drop in the soil.

Figure 9 shows the results of normal current density, over potential, metal voltage and axial current flowing along each well casing.

As expected, the normal current density on the well casing more distant from the anode (WC2) is significantly lower in comparison to the one closer (WC1). In addition, the over potential on the former is in average approximately $100 \mathrm{mV}$ lower than on the latter, except in the less conductive soil layer at depth between 1500 and $1600 \mathrm{~m}$ where the difference in over potential is negligible.

In this case none of the well casings show anodic behaviour but with different conditions this may occur and would be predicted by the model. 

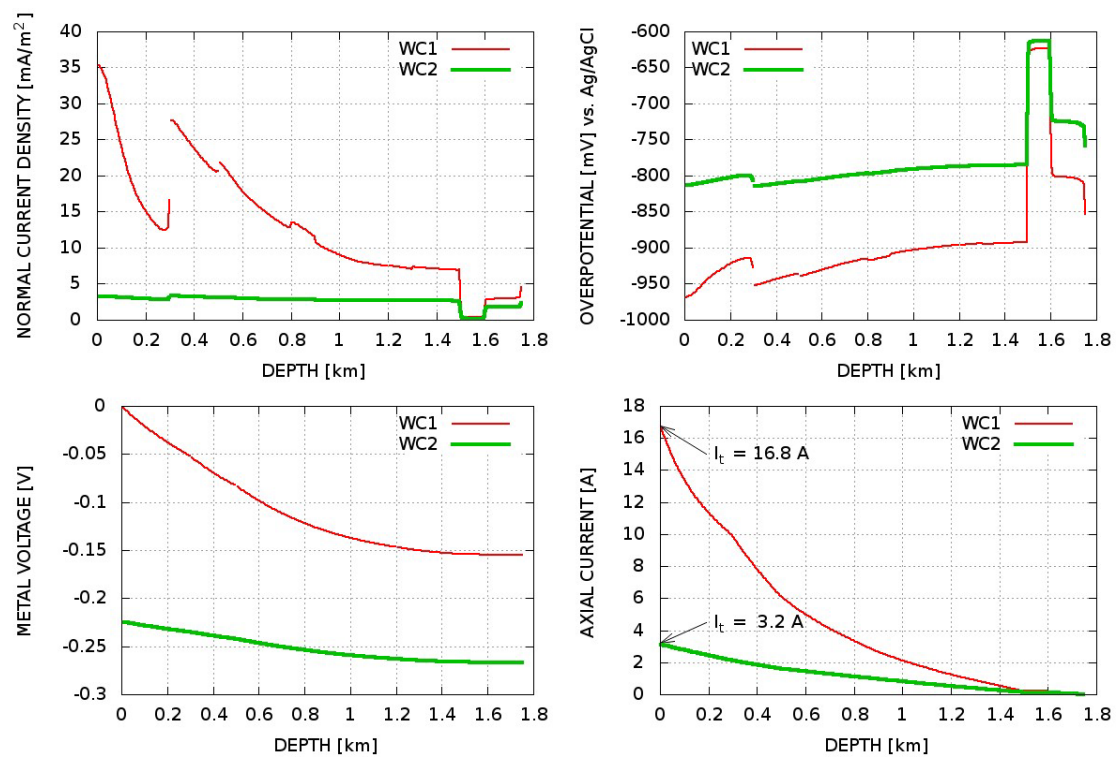

Figure 9: Comparison of normal current density, overpotential, metal voltage and total axial current for the two well casings connected to the ICCP anode.

\section{Conclusions}

A new approach for the numerical modelling of $\mathrm{CP}$ systems applied to well casing structures in vertically stratified soil has been developed. A significant advantage over the traditional multi-region technique is the reduction of the number of degrees of freedom. In addition the modelling preparation is simplified, as interfaces between soil layers do not need to be modelled at all.

The multilayer (ML) approach can easily represent thin layers of electrolyte without additional mesh discretisation thus substantially reducing the work of the user.

A feature of the ML is that the computational cost of the calculation does not increase with the number of layers. The results obtained with the ML are in good agreement with the traditional multi-domain approach.

The most important benefit of the ML approach is the simplicity in its application to well casing applications as the data can be prepared very easily and the calculations made within a few minutes. Consequently sensitivity studies on proposed new designs can be quickly completed.

The model can used to optimise the use of measurement techniques such as surface E-LogI measurements and CPET logs to improve the cost effectiveness and reliability of CP designs.

An approach for incorporating the cement that fills the annular space between the metal and the soil has been proposed and the influence of different qualities 
of the cement on the CP performance has been quantified. In cases where there is doubt about the quality of the cement in a particular layer the model can be used to assess its impact and identify effective corrosion control options.

The model has been applied to multiple well situations where there is the possibility of interference between the wells. Results have been presented where not only the ohmic drop in the electrolyte is considered but also the IR drop in the power supply cables, flow lines and other associated equipment and structures is modelled.

\section{References}

[1] C.A. Brebbia, J.C.F. Telles and L.C. Wrobel. Boundary Element Techniques - Theory and Application in Engineering. Springer Verlag Berlin, Heidelberg NY, Tokyo. 1984.

[2] Andres B Peratta, John M W Baynham, and Robert A. Adey . A Computational Approach for Assessing Coating Performance in Cathodically Protected Transmission Pipelines. CORROSION 2009, Paper 6595 Atlanta, Georgia. NACE International 2009.

[3] Andres B Peratta, John M W Baynham, and Robert A. Adey . Advances In Cathodic Protection Modelling of Deep Well Casings In Multi-Layered Media. CORROSION 2009, Paper 6555 Atlanta, Georgia. NACE International 2009.

[4] D. P. Riemer and M. E. Orazem, "Modelling Coating Flaws with NonLinear Polarization Curves for Long Pipelines," in Corrosion and Cathodic Protection Modelling and Simulation, Volume 12 of Advances in Boundary Elements, R. A. Adey, editor, WIT press, Southampton, 2005, 225-259.

[5] D. P. Riemer and M. E. Orazem, "Application of Boundary Element Models to Predict the Effectiveness of Coupons for Accessing Cathodic Protection of Buried Structures," Corrosion, 56 (2000) 794-800.

[6] R.A. Adey, J. Baynham. Design and optimization of cathodic protection systems using computer simulation. CORROSION 2000, Paper \ 723. Houston, Texas. NACE International, 2000.

[7] V. Seremet. Handbook of Green's Functions and Matrices. WIT Press Southampton, Boston. 2003

[8] H. Ymeri, B. Nauwelaers and K. Maex. Computation of conductance and capacitance for IC interconnects on a General Lossy Multilayer substrate. Active and Passive Elec. Comp. Vol 24. pp 87-114, 2001

[9] T. Smedes, N.P. van der Mejis and A.J. van Gendered. Boundary Element methods for 3D capacitance and substrate resistance calculations in inhomogeneous media in a VLSI layout verification package. Advances in Engineering Software 20. pp 19-27. 1994.

[10] M. Roche, J. Vittonato and M. Jebara. "Cathodic Protection Modelling of Deep Wells Casing by 3D software simulation: Comparison with E-Logl and CPET Data”. Paper 08273. NACE Corrosion 2008.

[11] J.D. Jackson. Classical Electrodynamics. 2nd ed. John Wiley \& Sons. NY, Chichester, Brisbane, Toronto, Singapore. 1975 